\title{
Iterative algorithm of common solutions for a constrained convex minimization problem, a quasi-variational inclusion problem and the fixed point problem of a strictly pseudo-contractive mapping
}

Yifen Ke and Changfeng Ma*

${ }^{*}$ Correspondence: macf@fjnu.edu.cn

School of Mathematics and Computer Science, Fujian Normal University, Fuzhou, 350007 P.R. China

\begin{abstract}
In this paper, a new iterative algorithm is proposed for finding a common solution to a constrained convex minimization problem, a quasi-variational inclusion problem and the fixed point problem of a strictly pseudo-contractive mapping in a real Hilbert space. It is proved that the sequence generated by the proposed algorithm converges strongly to a common solution of the three above described problems. By applying this result to some special cases, several interesting results can be obtained. MSC: 47H09; 47H10;49J40

Keywords: iterative algorithm; constrained convex minimization; quasi-variational inclusion; strictly pseudo-contractive; fixed point; convergence analysis
\end{abstract}

\section{Introduction}

Variational inequalities, introduced by Hartman and Stampacchia [1] in the early sixties, are one of the most interesting and intensively studied classes of mathematical problems. They are a very powerful tool of the current mathematical technology and have been extended to study a considerable amount of problems arising in mechanics, physics, optimization and control, nonlinear programming, transportation equilibrium and engineering sciences (see, e.g., [2-4]). As a useful and important generalization of variational inequalities, quasi-variational inclusion problems are also further studied (see, e.g., [5-14] and the references contained therein).

Throughout this paper, we assume that $H$ is a real Hilbert space with the inner product $\langle\cdot, \cdot\rangle$ and the induced norm $\|\cdot\|$, and let $C$ be a nonempty closed convex subset of $H . \mathcal{F}(T)$ denotes a fixed point set of the mapping $T$.

Let $\Phi$ be a single-valued mapping of $C$ into $H$ and $M$ be a multi-valued mapping with domain $\mathbb{D}(M)=C$. The quasi-variational inclusion problem is to find $u \in C$ such that

$$
0 \in \Phi(u)+M u
$$

\section{Springer}

○2014 Ke and Ma; licensee Springer. This is an Open Access article distributed under the terms of the Creative Commons Attribution License (http://creativecommons.org/licenses/by/2.0), which permits unrestricted use, distribution, and reproduction in any medium, provided the original work is properly cited. 
The solution set of quasi-variational inclusion problem (1.1) is denoted by $\operatorname{VI}(C, \Phi, M)$. In particular, if $M=\partial \delta_{C}$, where $\delta_{C}: H \rightarrow[0, \infty]$ is the indicator function of $C$, i.e.,

$$
\delta_{C}(x)= \begin{cases}0, & x \in C, \\ +\infty, & x \notin C,\end{cases}
$$

then the variational inclusion problem (1.1) is equivalent to finding $u \in C$ such that

$$
\langle\Phi(u), v-u\rangle \geq 0, \quad \forall v \in C
$$

This problem is called the Hartman-Stampacchia variational inequality problem [1]. The solution set of problem (1.2) is denoted by $\mathrm{VI}(C, \Phi)$.

Recall that $T: C \rightarrow C$ is called a $k$-strictly pseudo-contractive mapping if there exists a constant $k \in[0,1)$ such that

$$
\|T x-T y\|^{2} \leq\|x-y\|^{2}+k\|(I-T) x-(I-T) y\|^{2}, \quad \forall x, y \in C,
$$

and $T$ is called a pseudo-contractive mapping if

$$
\|T x-T y\|^{2} \leq\|x-y\|^{2}+\|(I-T) x-(I-T) y\|^{2}, \quad \forall x, y \in C .
$$

It is obvious that $k=0$, then the mapping $T$ is nonexpansive, that is,

$$
\|T x-T y\| \leq\|x-y\|, \quad \forall x, y \in C .
$$

It is well known that finding fixed points of nonexpansive mappings is an important topic in the theory of nonexpansive mappings and has wide applications in a number of applied areas, such as the convex feasibility problem $[15,16]$, the split feasibility problem [17], image recovery and signal processing [18]. After that, as an important generalization of nonexpansive mappings, strictly pseudo-contractive mappings become one of the most interesting studied classes of nonexpansive mappings (see, e.g., [19-22]). In fact, strictly pseudo-contractive mappings have more powerful applications than nonexpansive mappings do such as in solving an inverse problem [23].

In order to find a common element of the solution set of quasi-variational inclusion problem (1.1) and the fixed point set of $k$-strictly pseudo-contractive mapping (1.3), which is also a solution of the following constrained convex minimization problem:

$$
\min _{x \in C} f(x)
$$

where $f: C \rightarrow R$ is a real-valued convex function and assumes that problem (1.4) is consistent (i.e., its solution set is nonempty), let $\Omega$ denote its solution set. Ceng et al. [24] 
studied the following algorithm: take $x_{0}=x \in C$ arbitrarily and

$$
\left\{\begin{aligned}
y_{n} & =P_{C}\left(x_{n}-\lambda_{n} \nabla f\left(x_{n}\right)\right), \\
t_{n} & =P_{C}\left(x_{n}-\lambda_{n} \nabla f\left(y_{n}\right)\right), \\
z_{n} & =\left(1-\alpha_{n}-\widehat{\alpha}_{n}\right) x_{n}+\alpha_{n} J_{M, \mu_{n}}\left(t_{n}-\mu_{n} \Phi\left(t_{n}\right)\right) \\
& +\widehat{\alpha}_{n} S J_{M, \mu_{n}}\left(t_{n}-\mu_{n} \Phi\left(t_{n}\right)\right), \\
C_{n} & =\left\{z \in C:\left\|z_{n}-z\right\| \leq\left\|x_{n}-z\right\|\right\}, \\
Q_{n} & =\left\{z \in C:\left\langle x_{n}-z, x-x_{n}\right\rangle \geq 0\right\}, \\
x_{n+1} & =P_{C_{n} \cap Q_{n}} x, \quad n \geq 0 .
\end{aligned}\right.
$$

Under appropriate conditions they obtained one strong convergence theorem.

In this paper, motivated and inspired by the above facts, we propose a new algorithm as follows: take $x_{0} \in C$ arbitrarily, set $C_{0}=C$, and

$$
\left\{\begin{array}{l}
y_{n}=P_{C}\left(x_{n}-\lambda_{n} \nabla f\left(x_{n}\right)\right), \\
z_{n}=P_{C}\left(x_{n}-\lambda_{n} \nabla f\left(y_{n}\right)\right), \\
t_{n}=J_{M, \mu_{n}}\left(z_{n}-\mu_{n} \Phi\left(z_{n}\right)\right), \\
w_{n}=\alpha_{n} t_{n}+\left(1-\alpha_{n}\right) S t_{n}, \\
C_{n+1}=\left\{w \in C_{n}:\left\|w_{n}-w\right\| \leq\left\|x_{n}-w\right\|\right\}, \\
x_{n+1}=P_{C_{n+1}} x_{0}, \quad n \geq 0,
\end{array}\right.
$$

and also get a strong convergence theorem under certain conditions.

The remainder of this paper is organized as follows. In Section 2, some definitions and lemmas are provided to get the main results of this paper. In Section 3, we give and prove one strong convergence theorem about our proposed algorithm. Finally, in Section 4, we apply our conclusion to some special cases.

\section{Preliminaries}

Let $H$ be a real Hilbert space. It is well known that

$$
\|x-y\|^{2}=\|x\|^{2}-2\langle x, y\rangle+\|y\|^{2}
$$

and

$$
\|t x+(1-t) y\|^{2}=t\|x\|^{2}+(1-t)\|y\|^{2}-t(1-t)\|x-y\|^{2}
$$

for all $x, y \in H$ and $t \in[0,1]$.

Now, we recall some definitions and useful results which will be used in the next section.

Definition 2.1 Let $T: C \rightarrow H$ be a nonlinear operator.

(1) $T$ is Lipschitz continuous if there exists a constant $L>0$ such that

$$
\|T x-T y\| \leq L\|x-y\|, \quad \forall x, y \in C .
$$

(2) $T$ is monotone if

$$
\langle T x-T y, x-y\rangle \geq 0, \quad \forall x, y \in C .
$$


(3) $T$ is $\rho$-strongly monotone if there exists a number $\rho>0$ such that

$$
\langle T x-T y, x-y\rangle \geq \rho\|x-y\|^{2}, \quad \forall x, y \in C .
$$

(4) $T$ is $\eta$-inverse-strongly monotone if there exists a number $\eta>0$ such that

$$
\langle T x-T y, x-y\rangle \geq \eta\|T x-T y\|^{2}, \quad \forall x, y \in C .
$$

It is easy to see that the following results hold: (i) strongly monotone is monotone; (ii) an $\eta$-inverse-strongly monotone mapping is monotone and $\frac{1}{\eta}$-Lipschitz continuous; (iii) $T$ is $k$-strictly pseudo-contractive, then $I-T$ is $\frac{1-k}{2}$-inverse strongly monotone.

Definition 2.2 A multi-valued mapping $M: \mathbb{D}(M) \subset H \rightarrow 2^{H}$ is called monotone if its graph $G(M)=\{(x, f) \in H \times H: x \in \mathbb{D}(M), f \in M x\}$ is a monotone set in $H \times H$, that is, $M$ is monotone if and only if

$$
(x, f),(y, g) \in G(M) \quad \Rightarrow \quad\langle x-y, f-g\rangle \geq 0 .
$$

A monotone multi-valued mapping $M$ is called maximal if for any $(x, f) \in H \times H,\langle x-y, f-$ $g\rangle \geq 0$ for every $(y, g) \in G(M)$ implies $f \in M x$.

Remark 2.1 [24] The following results are equivalent:

(1) A multi-valued mapping $M$ is maximal monotone;

(2) $M$ is monotone and $(I+\lambda M) \mathbb{D}(M)=H$ for each $\lambda>0$;

(3) $M$ is monotone and its graph $G(M)$ is not properly contained in the graph of any other monotone mapping in $H$.

Definition 2.3 $P_{C}: H \rightarrow C$ is called a metric projection if for every point $x \in H$, there exists a unique nearest point in $C$, denoted by $P_{C} x$, such that

$$
\left\|x-P_{C} x\right\| \leq\|x-y\|, \quad \forall y \in C .
$$

Lemma 2.1 Let $C$ be a nonempty closed convex subset of $H$ and let $P_{C}: H \rightarrow C$ be a metric projection, then

(1) $\left\|P_{C} x-P_{C} y\right\|^{2} \leq\left\langle x-y, P_{C} x-P_{C} y\right\rangle, \forall x, y \in H$;

(2) moreover, $P_{C}$ is a nonexpansive mapping, i.e., $\left\|P_{C} x-P_{C} y\right\| \leq\|x-y\|, \forall x, y \in H$;

(3) $\left\langle x-P_{C} x, y-P_{C} x\right\rangle \leq 0, \forall x \in H, y \in C$;

(4) $\|x-y\|^{2} \geq\left\|x-P_{C} x\right\|^{2}+\left\|y-P_{C} x\right\|^{2}, \forall x \in H, y \in C$.

Definition 2.4 Let $M: \mathbb{D}(M) \subset H \rightarrow 2^{H}$ be a multi-valued maximal monotone mapping, then the single-valued mapping $J_{M, \mu}: H \rightarrow H$ defined by

$$
J_{M, \mu} x=(I+\mu M)^{-1} x, \quad \forall x \in H
$$

is called the resolvent operator associated with $M$, where $\mu$ is any positive number and $I$ is the identity mapping. 
Lemma $2.2[5,24,25]$ The resolvent operator $J_{M, \mu}$ associated with $M$ is single-valued and firmly nonexpansive, i.e.,

$$
\left\|J_{M, \mu} x-J_{M, \mu} y\right\|^{2} \leq\left\langle J_{M, \mu} x-J_{M, \mu} y, x-y\right\rangle, \quad \forall x, y \in H .
$$

Consequently, $J_{M, \mu}$ is nonexpansive and monotone.

Lemma 2.3 [24] Let $M$ be a multi-valued maximal monotone mapping with $\mathbb{D}(M)=C$. Then, for any given $\mu>0, u \in C$ is a solution of problem (1.1) if and only if $u \in C$ satisfies

$$
u=J_{M, \mu}(u-\mu \Phi(u)) \text {. }
$$

Lemma 2.4 [24] Let $M$ be a multi-valued maximal monotone mapping with $\mathbb{D}(M)=C$ and let $\Phi: C \rightarrow H$ be a monotone, continuous and single-valued mapping. Then $M+\Phi$ is maximal monotone.

In the sequel, we use $x_{n} \rightarrow x$ and $x_{n} \rightarrow x$ to denote the weak convergence and strong convergence of the sequence $\left\{x_{n}\right\}$ in $H$, respectively.

Lemma 2.5 [26] Let $C$ be a nonempty closed convex subset of $H$ and let $T: C \rightarrow C$ be a $k$-strictly pseudocontractive mapping, then the following results hold:

(1) inequality (1.3) is equivalent to

$$
\langle T x-T y, x-y\rangle \leq\|x-y\|^{2}-\frac{1-k}{2}\|(I-T) x-(I-T) y\|^{2}, \quad \forall x, y \in C .
$$

(2) $T$ is Lipschitz continuous with a constant $\frac{1+k}{1-k}$, i.e.,

$$
\|T x-T y\| \leq \frac{1+k}{1-k}\|x-y\|, \quad \forall x, y \in C
$$

(3) (Demi-closedness principle) $I-T$ is demi-closed on $C$, that is,

$$
\text { if } x_{n} \rightarrow x^{*} \in C \text { and }(I-T) x_{n} \rightarrow 0 \text {, then } x^{*}=T x^{*} .
$$

Lemma 2.6 Let $C$ be a nonempty closed convex subset of $H$ and let $T: C \rightarrow H$ be an $\eta$ inverse-strongly monotone mapping, then for all $x, y \in C$ and $\eta>0$, we have

$$
\begin{aligned}
\|(I-\lambda T) x-(I-\lambda T) y\|^{2} & =\|(x-y)-\lambda(T x-T y)\|^{2} \\
& =\|x-y\|^{2}-2 \lambda\langle T x-T y, x-y\rangle+\lambda^{2}\|T x-T y\|^{2} \\
& \leq\|x-y\|^{2}+\lambda(\lambda-2 \eta)\|T x-T y\|^{2} .
\end{aligned}
$$

So, if $0<\lambda \leq 2 \eta$, then $I-\lambda T$ is a nonexpansive mapping from $C$ to $H$.

Lemma $2.7[24,27]$ Let $A: C \rightarrow H$ be a monotone, Lipschitz continuous mapping, and let $N_{C} v$ be the normal cone to $C$ at $v \in C$, i.e.,

$$
N_{C} v=\{z \in H:\langle v-u, z\rangle \geq 0, \forall u \in C\} .
$$


Define

$$
T \nu= \begin{cases}A v+N_{C} v, & v \in C, \\ \emptyset, & v \notin C .\end{cases}
$$

Then, $T$ is maximal monotone and $0 \in T v$ if and only if $v \in \mathrm{VI}(C, A)$.

For the minimization problem (1.4), if $f$ is (Frechet) differentiable, then we have the following lemma.

Lemma 2.8 [28] (Optimality condition) A necessary condition of optimality for a point $x^{*} \in C$ to be a solution of the minimization problem (1.4) is that $x^{*}$ solves the variational inequality

$$
\left\langle\nabla f\left(x^{*}\right), x-x^{*}\right\rangle \geq 0, \quad \forall x \in C
$$

Equivalently, $x^{*} \in C$ solves the fixed point equation

$$
x^{*}=P_{C}\left(x^{*}-\lambda \nabla f\left(x^{*}\right)\right)
$$

for every constant $\lambda>0$. If, in addition, $f$ is convex, then the optimality condition (2.1) is also sufficient.

\section{Main results}

In this section, we prove a strong convergence theorem by an iterative algorithm for finding a solution of the constrained convex minimization problem (1.4), which is also a common solution of the quasi-variational inclusion problem (1.1) and the fixed point problem of a $k$-strictly pseudo-contractive mapping (1.3) in a real Hilbert space.

Theorem 3.1 Let $C$ be a nonempty closed convex subset of a real Hilbert space H. For the minimization problem (1.4), assume that $f$ is (Frechet) differentiable and the gradient $\nabla f$ is a $\rho$-inverse-strongly monotone mapping. Let $\Phi: C \rightarrow H$ be an $\eta$-inverse-strongly monotone mapping and $M$ be a maximal monotone mapping with $\mathbb{D}(M)=C$, and let $S: C \rightarrow C$ be a $k$-strictly pseudo-contractive mapping such that $\mathcal{F}=\mathcal{F}(S) \cap \Omega \cap \mathrm{VI}(C, \Phi, M) \neq \emptyset$. Pick any $x_{0} \in C$ and set $C_{0}=C$. Let $\left\{x_{n}\right\} \subset C$ be a sequence generated by

$$
\left\{\begin{array}{l}
y_{n}=P_{C}\left(x_{n}-\lambda_{n} \nabla f\left(x_{n}\right)\right), \\
z_{n}=P_{C}\left(x_{n}-\lambda_{n} \nabla f\left(y_{n}\right)\right), \\
t_{n}=J_{M, \mu_{n}}\left(z_{n}-\mu_{n} \Phi\left(z_{n}\right)\right), \\
w_{n}=\alpha_{n} t_{n}+\left(1-\alpha_{n}\right) S t_{n}, \\
C_{n+1}=\left\{w \in C_{n}:\left\|w_{n}-w\right\| \leq\left\|x_{n}-w\right\|\right\}, \\
x_{n+1}=P_{C_{n+1}} x_{0}, \quad n \geq 0,
\end{array}\right.
$$

where the following conditions hold:

(i) $0<\liminf _{n \rightarrow \infty} \lambda_{n} \leq \limsup _{n \rightarrow \infty} \lambda_{n}<\rho$;

(ii) $\epsilon \leq \mu_{n} \leq 2 \eta$ for some $\epsilon \in(0,2 \eta]$;

(iii) $k<\liminf _{n \rightarrow \infty} \alpha_{n} \leq \limsup _{n \rightarrow \infty} \alpha_{n}<1$.

Then the sequence $\left\{x_{n}\right\}$ converges strongly to $P_{\mathcal{F}} x_{0}$. 
Proof It is obvious that $C_{n}$ is closed for each $n \in \mathbb{N}$. Since $\left\|w_{n}-w\right\| \leq\left\|x_{n}-w\right\|$ is equivalent to

$$
\left\|w_{n}-x_{n}\right\|^{2}+2\left\langle w_{n}-x_{n}, x_{n}-w\right\rangle \leq 0,
$$

we have that $C_{n}$ is convex for each $n \in \mathbb{N}$. Therefore, $\left\{x_{n}\right\}$ is well defined. We divide the proof into five steps.

Step 1 . We show by induction that $\mathcal{F} \subset C_{n}$ for each $n \in \mathbb{N}$.

It is obvious that $\mathcal{F} \subset C_{0}=C$. Suppose that $\mathcal{F} \subset C_{n}$ for some $n \in \mathbb{N}$. Let $p \in \mathcal{F}$, we have

$$
\begin{aligned}
\left\|w_{n}-p\right\|^{2} & =\left\|\alpha_{n} t_{n}+\left(1-\alpha_{n}\right) S t_{n}-p\right\|^{2} \\
& =\left\|\alpha_{n}\left(t_{n}-p\right)+\left(1-\alpha_{n}\right)\left(S t_{n}-p\right)\right\|^{2} \\
& =\alpha_{n}\left\|t_{n}-p\right\|^{2}+\left(1-\alpha_{n}\right)\left\|S t_{n}-p\right\|^{2}-\alpha_{n}\left(1-\alpha_{n}\right)\left\|t_{n}-S t_{n}\right\|^{2} \\
& \leq \alpha_{n}\left\|t_{n}-p\right\|^{2}+\left(1-\alpha_{n}\right)\left[\left\|t_{n}-p\right\|^{2}+k\left\|t_{n}-S t_{n}\right\|^{2}\right]-\alpha_{n}\left(1-\alpha_{n}\right)\left\|t_{n}-S t_{n}\right\|^{2} \\
& =\left\|t_{n}-p\right\|^{2}+\left(1-\alpha_{n}\right)\left(k-\alpha_{n}\right)\left\|t_{n}-S t_{n}\right\|^{2} \\
& \leq\left\|t_{n}-p\right\|^{2} .
\end{aligned}
$$

According to Lemma 2.3, Lemma 2.2 and Lemma 2.6, we get

$$
\begin{aligned}
\left\|t_{n}-p\right\|^{2} & =\left\|J_{M, \mu_{n}}\left(z_{n}-\mu_{n} \Phi\left(z_{n}\right)\right)-J_{M, \mu_{n}}\left(p-\mu_{n} \Phi(p)\right)\right\|^{2} \\
& \leq\left\|z_{n}-\mu_{n} \Phi\left(z_{n}\right)-\left(p-\mu_{n} \Phi(p)\right)\right\|^{2} \\
& \leq\left\|z_{n}-p\right\|^{2}+\mu_{n}\left(\mu_{n}-2 \eta\right)\left\|\Phi\left(z_{n}\right)-\Phi(p)\right\|^{2} \\
& \leq\left\|z_{n}-p\right\|^{2} .
\end{aligned}
$$

Since the gradient $\nabla f$ is a $\rho$-inverse-strongly monotone mapping and $p \in \mathcal{F} \subset \Omega$, from Lemma 2.8, we have

$$
\left\langle\nabla f\left(y_{n}\right)-\nabla f(p), y_{n}-p\right\rangle \geq 0 \text { and }\left\langle\nabla f(p), y_{n}-p\right\rangle \geq 0 .
$$

From Lemma 2.1(4) and (3.4), we obtain

$$
\begin{aligned}
\left\|z_{n}-p\right\|^{2}= & \left\|P_{C}\left(x_{n}-\lambda_{n} \nabla f\left(y_{n}\right)\right)-p\right\|^{2} \\
\leq & \left\|x_{n}-\lambda_{n} \nabla f\left(y_{n}\right)-p\right\|^{2}-\left\|x_{n}-\lambda_{n} \nabla f\left(y_{n}\right)-z_{n}\right\|^{2} \\
= & \left\|x_{n}-p\right\|^{2}-\left\|x_{n}-z_{n}\right\|^{2}+2 \lambda_{n}\left\langle\nabla f\left(y_{n}\right), p-z_{n}\right\rangle \\
= & \left\|x_{n}-p\right\|^{2}-\left\|x_{n}-z_{n}\right\|^{2}+2 \lambda_{n}\left[\left\langle\nabla f\left(y_{n}\right)-\nabla f(p), p-y_{n}\right\rangle\right. \\
& \left.+\left\langle\nabla f(p), p-y_{n}\right\rangle+\left\langle\nabla f\left(y_{n}\right), y_{n}-z_{n}\right\rangle\right] \\
\leq & \left\|x_{n}-p\right\|^{2}-\left\|x_{n}-z_{n}\right\|^{2}+2 \lambda_{n}\left\langle\nabla f\left(y_{n}\right), y_{n}-z_{n}\right\rangle \\
= & \left\|x_{n}-p\right\|^{2}-\left\|x_{n}-y_{n}\right\|^{2}-2\left\langle x_{n}-y_{n}, y_{n}-z_{n}\right\rangle \\
& -\left\|y_{n}-z_{n}\right\|^{2}+2 \lambda_{n}\left\langle\nabla f\left(y_{n}\right), y_{n}-z_{n}\right\rangle
\end{aligned}
$$




$$
\begin{aligned}
= & \left\|x_{n}-p\right\|^{2}-\left\|x_{n}-y_{n}\right\|^{2}-\left\|y_{n}-z_{n}\right\|^{2} \\
& +2\left\langle x_{n}-\lambda_{n} \nabla f\left(y_{n}\right)-y_{n}, z_{n}-y_{n}\right\rangle .
\end{aligned}
$$

It is easy to see that $\rho$-inverse-strongly monotone mapping $\nabla f$ is $\frac{1}{\rho}$-Lipschitz continuous. Further, since $y_{n}=P_{C}\left(x_{n}-\lambda_{n} \nabla f\left(x_{n}\right)\right)$ and by Lemma 2.1(3) we have

$$
\begin{aligned}
\left\langle x_{n}\right. & \left.-\lambda_{n} \nabla f\left(y_{n}\right)-y_{n}, z_{n}-y_{n}\right\rangle \\
& =\left\langle x_{n}-\lambda_{n} \nabla f\left(x_{n}\right)-y_{n}, z_{n}-y_{n}\right\rangle+\left\langle\lambda_{n} \nabla f\left(x_{n}\right)-\lambda_{n} \nabla f\left(y_{n}\right), z_{n}-y_{n}\right\rangle \\
& \leq\left\langle\lambda_{n} \nabla f\left(x_{n}\right)-\lambda_{n} \nabla f\left(y_{n}\right), z_{n}-y_{n}\right\rangle \\
& \leq \lambda_{n}\left\|\nabla f\left(x_{n}\right)-\nabla f\left(y_{n}\right)\right\|\left\|z_{n}-y_{n}\right\| \\
& \leq \frac{\lambda_{n}}{\rho}\left\|x_{n}-y_{n}\right\|\left\|z_{n}-y_{n}\right\| .
\end{aligned}
$$

Substituting (3.6) into (3.5), we obtain

$$
\begin{aligned}
\left\|z_{n}-p\right\|^{2} \leq & \left\|x_{n}-p\right\|^{2}-\left\|x_{n}-y_{n}\right\|^{2}-\left\|y_{n}-z_{n}\right\|^{2} \\
& +2\left\langle x_{n}-\lambda_{n} \nabla f\left(y_{n}\right)-y_{n}, z_{n}-y_{n}\right\rangle \\
\leq & \left\|x_{n}-p\right\|^{2}-\left\|x_{n}-y_{n}\right\|^{2}-\left\|y_{n}-z_{n}\right\|^{2}+\frac{2 \lambda_{n}}{\rho}\left\|x_{n}-y_{n}\right\|\left\|z_{n}-y_{n}\right\| \\
\leq & \left\|x_{n}-p\right\|^{2}-\left\|x_{n}-y_{n}\right\|^{2}-\left\|y_{n}-z_{n}\right\|^{2}+\frac{\lambda_{n}^{2}}{\rho^{2}}\left\|x_{n}-y_{n}\right\|^{2}+\left\|z_{n}-y_{n}\right\|^{2} \\
= & \left\|x_{n}-p\right\|^{2}+\left(\frac{\lambda_{n}^{2}}{\rho^{2}}-1\right)\left\|x_{n}-y_{n}\right\|^{2} \\
\leq & \left\|x_{n}-p\right\|^{2} .
\end{aligned}
$$

From (3.2), (3.3) and (3.7), we have

$$
\left\|w_{n}-p\right\| \leq\left\|x_{n}-p\right\|
$$

Hence $p \in C_{n+1}$. This implies that $p \in C_{n}$ for all $n \in \mathbb{N}$.

Step 2. We prove that $\lim _{n \rightarrow \infty}\left\|x_{n+1}-x_{n}\right\|=0$ and $\lim _{n \rightarrow \infty}\left\|x_{n}-w_{n}\right\|=0$.

Let $x^{*}=P_{\mathcal{F}} x_{0}$. From $x_{n}=P_{C_{n}} x_{0}$ and $x^{*} \in \mathcal{F} \subset C_{n}$, we obtain

$$
\left\|x_{n}-x_{0}\right\| \leq\left\|x^{*}-x_{0}\right\|
$$

Then $\left\{x_{n}\right\}$ is bounded. This implies that $\left\{z_{n}\right\},\left\{t_{n}\right\}$ and $\left\{w_{n}\right\}$ are also bounded. Since $x_{n}=$ $P_{C_{n}} x_{0}$ and $x_{n+1} \in C_{n+1} \subset C_{n}$, we have

$$
\left\|x_{n}-x_{0}\right\| \leq\left\|x_{n+1}-x_{0}\right\|
$$

Therefore $\lim _{n \rightarrow \infty}\left\|x_{n}-x_{0}\right\|$ exists. From $x_{n}=P_{C_{n}} x_{0}, x_{n+1} \in C_{n+1} \subset C_{n}$ and Lemma 2.1(4), we obtain

$$
0 \leq\left\|x_{n+1}-x_{n}\right\|^{2} \leq\left\|x_{0}-x_{n+1}\right\|^{2}-\left\|x_{0}-x_{n}\right\|^{2},
$$


which implies

$$
\lim _{n \rightarrow \infty}\left\|x_{n+1}-x_{n}\right\|=0
$$

It follows from $x_{n+1} \in C_{n+1}$ that $\left\|w_{n}-x_{n+1}\right\| \leq\left\|x_{n}-x_{n+1}\right\|$, and hence

$$
\left\|x_{n}-w_{n}\right\| \leq\left\|x_{n}-x_{n+1}\right\|+\left\|x_{n+1}-w_{n}\right\| \leq 2\left\|x_{n}-x_{n+1}\right\| .
$$

From (3.10) and (3.11), we have

$$
\lim _{n \rightarrow \infty}\left\|x_{n}-w_{n}\right\|=0
$$

Step 3. We show that $\lim _{n \rightarrow \infty}\left\|t_{n}-S t_{n}\right\|=0, \lim _{n \rightarrow \infty}\left\|x_{n}-z_{n}\right\|=0$ and $\lim _{n \rightarrow \infty} \| x_{n}-$ $t_{n} \|=0$.

For $p \in \mathcal{F}$, from (3.2), (3.3) and (3.7), we have

$$
\begin{aligned}
\left\|w_{n}-p\right\|^{2} & \leq\left\|t_{n}-p\right\|^{2}+\left(1-\alpha_{n}\right)\left(k-\alpha_{n}\right)\left\|t_{n}-S t_{n}\right\|^{2} \\
& \leq\left\|z_{n}-p\right\|^{2}+\left(1-\alpha_{n}\right)\left(k-\alpha_{n}\right)\left\|t_{n}-S t_{n}\right\|^{2} \\
& \leq\left\|x_{n}-p\right\|^{2}+\left(\frac{\lambda_{n}^{2}}{\rho^{2}}-1\right)\left\|x_{n}-y_{n}\right\|^{2}+\left(1-\alpha_{n}\right)\left(k-\alpha_{n}\right)\left\|t_{n}-S t_{n}\right\|^{2} .
\end{aligned}
$$

Then

$$
\begin{aligned}
& \left(1-\frac{\lambda_{n}^{2}}{\rho^{2}}\right)\left\|x_{n}-y_{n}\right\|^{2}+\left(1-\alpha_{n}\right)\left(\alpha_{n}-k\right)\left\|t_{n}-S t_{n}\right\|^{2} \\
& \quad \leq\left\|x_{n}-p\right\|^{2}-\left\|w_{n}-p\right\|^{2} \\
& \quad \leq\left(\left\|x_{n}-p\right\|+\left\|w_{n}-p\right\|\right)\left\|x_{n}-w_{n}\right\| .
\end{aligned}
$$

Since $0<\liminf _{n \rightarrow \infty} \lambda_{n} \leq \lim \sup _{n \rightarrow \infty} \lambda_{n}<\rho$ and $k<\liminf _{n \rightarrow \infty} \alpha_{n} \leq \limsup _{n \rightarrow \infty} \alpha_{n}<1$, from (3.12) and (3.13) we get

$$
\lim _{n \rightarrow \infty}\left\|x_{n}-y_{n}\right\|=0
$$

and

$$
\lim _{n \rightarrow \infty}\left\|t_{n}-S t_{n}\right\|=0
$$

As $\nabla f$ is $\frac{1}{\rho}$-Lipschitz continuous, we have

$$
\begin{aligned}
\left\|z_{n}-y_{n}\right\| & =\left\|P_{C}\left(x_{n}-\lambda_{n} \nabla f\left(y_{n}\right)\right)-P_{C}\left(x_{n}-\lambda_{n} \nabla f\left(x_{n}\right)\right)\right\| \\
& \leq\left\|x_{n}-\lambda_{n} \nabla f\left(y_{n}\right)-\left(x_{n}-\lambda_{n} \nabla f\left(x_{n}\right)\right)\right\| \\
& =\lambda_{n}\left\|\nabla f\left(y_{n}\right)-\nabla f\left(x_{n}\right)\right\| \\
& \leq \frac{\lambda_{n}}{\rho}\left\|x_{n}-y_{n}\right\| .
\end{aligned}
$$


Hence

$$
\lim _{n \rightarrow \infty}\left\|z_{n}-y_{n}\right\|=0
$$

From (3.14) and (3.16), we obtain

$$
\lim _{n \rightarrow \infty}\left\|x_{n}-z_{n}\right\|=0
$$

We observe

$$
\begin{aligned}
\left\|w_{n}-t_{n}\right\| & =\left\|\alpha_{n} t_{n}+\left(1-\alpha_{n}\right) S t_{n}-t_{n}\right\| \\
& =\left(1-\alpha_{n}\right)\left\|S t_{n}-t_{n}\right\| \\
& \leq\left\|S t_{n}-t_{n}\right\| .
\end{aligned}
$$

From (3.15), we get

$$
\lim _{n \rightarrow \infty}\left\|w_{n}-t_{n}\right\|=0
$$

Combining (3.12) and (3.19), we have

$$
\lim _{n \rightarrow \infty}\left\|x_{n}-t_{n}\right\|=0
$$

Step 4 . Since $\left\{x_{n}\right\}$ is bounded, there exists a subsequence $\left\{x_{n_{i}}\right\}$ which converges weakly to $u$. We show that

$$
u \in \mathcal{F}=\mathcal{F}(S) \cap \Omega \cap \operatorname{VI}(C, \Phi, M) .
$$

Indeed, firstly, we show $u \in \mathcal{F}(S)$. Since $\left\|x_{n}-t_{n}\right\| \rightarrow 0$ and $x_{n_{i}} \rightarrow u$, we have $t_{n_{i}} \rightarrow u$. From $\left\|S t_{n}-t_{n}\right\| \rightarrow 0$, we obtain $\left\|S t_{n_{i}}-t_{n_{i}}\right\| \rightarrow 0$ as $i \rightarrow \infty$. By Lemma 2.5 (Demiclosedness principle), we can conclude that $u \in \mathcal{F}(S)$.

Secondly, we show $u \in \Omega$. Since $z_{n}=P_{C}\left(x_{n}-\lambda_{n} \nabla f\left(y_{n}\right)\right)$ and by Lemma 2.1(3), we have

$$
\left\langle x_{n}-\lambda_{n} \nabla f\left(y_{n}\right)-z_{n}, z_{n}-v\right\rangle \geq 0,
$$

that is,

$$
\left\langle v-z_{n}, \frac{z_{n}-x_{n}}{\lambda_{n}}+\nabla f\left(y_{n}\right)\right\rangle \geq 0 .
$$

Let

$$
T v= \begin{cases}\nabla f(v)+N_{C} v, & v \in C, \\ \emptyset, & v \notin C .\end{cases}
$$

Then, from Lemma 2.7, we know that $T$ is maximal monotone and $0 \in T v$ if and only if $v \in \operatorname{VI}(C, \nabla f)$. Let $G(T)$ be the graph of $T$ and let $(v, h) \in G(T)$. Then we have $h \in T v=$ 
$\nabla f(v)+N_{C} v$. Hence $h-\nabla f(v) \in N_{C} v$. So, we have

$$
\langle v-z, h-\nabla f(v)| \geq 0, \quad \forall z \in C
$$

Therefore,

$$
\begin{aligned}
& \left\langle v-z_{n_{i}}, h\right\rangle \\
& \quad \geq\left\langle v-z_{n_{i}}, \nabla f(v)\right\rangle \\
& \quad \geq\left\langle v-z_{n_{i}}, \nabla f(v)\right\rangle-\left\langle v-z_{n_{i}}, \frac{z_{n_{i}}-x_{n_{i}}}{\lambda_{n_{i}}}+\nabla f\left(y_{n_{i}}\right)\right\rangle \\
& \quad=\left\langle v-z_{n_{i}}, \nabla f(v)-\nabla f\left(z_{n_{i}}\right)\right\rangle+\left\langle v-z_{n_{i}}, \nabla f\left(z_{n_{i}}\right)-\nabla f\left(y_{n_{i}}\right)\right\rangle-\left\langle v-z_{n_{i}}, \frac{z_{n_{i}}-x_{n_{i}}}{\lambda_{n_{i}}}\right\rangle \\
& \quad \geq\left\langle v-z_{n_{i}}, \nabla f\left(z_{n_{i}}\right)-\nabla f\left(y_{n_{i}}\right)\right\rangle-\left\langle v-z_{n_{i}}, \frac{z_{n_{i}}-x_{n_{i}}}{\lambda_{n_{i}}}\right\rangle .
\end{aligned}
$$

Since $\left\|x_{n}-y_{n}\right\| \rightarrow 0$ and $\left\|x_{n}-z_{n}\right\| \rightarrow 0$, we have $y_{n_{i}} \rightarrow u$ and $z_{n_{i}} \rightarrow u$. Then, from (3.21), we obtain $\langle v-u, h-0\rangle=\langle v-u, h\rangle \geq 0$ as $i \rightarrow \infty$. Since $T$ is maximal monotone, we have $0 \in T u$ and hence $u \in \operatorname{VI}(C, \nabla f)$. According to Lemma 2.8, we obtain $u \in \Omega$.

Finally, let us show $u \in \operatorname{VI}(C, \Phi, M)$. Since $\Phi: C \rightarrow H$ is $\eta$-inverse-strongly monotone and $M$ is maximal monotone, by Lemma 2.4 we know that $M+\Phi$ is maximal monotone. Take a fixed $(y, g) \in G(M+\Phi)$ arbitrarily. Then we have $g \in \Phi(y)+M y$, that is,

$$
g-\Phi(y) \in M y
$$

Since $t_{n_{i}}=J_{M, \mu_{n_{i}}}\left(z_{n_{i}}-\mu_{n_{i}} \Phi\left(z_{n_{i}}\right)\right)$, then

$$
\frac{1}{\mu_{n_{i}}}\left(z_{n_{i}}-\mu_{n_{i}} \Phi\left(z_{n_{i}}\right)-t_{n_{i}}\right) \in M t_{n_{i}}
$$

Therefore,

$$
\left\langle y-t_{n_{i}}, g-\Phi(y)-\frac{1}{\mu_{n_{i}}}\left(z_{n_{i}}-\mu_{n_{i}} \Phi\left(z_{n_{i}}\right)-t_{n_{i}}\right)\right\rangle \geq 0,
$$

which hence yields

$$
\begin{aligned}
\langle y & \left.-t_{n_{i}}, g\right\rangle \\
& \geq\left\langle y-t_{n_{i}}, \Phi(y)+\frac{1}{\mu_{n_{i}}}\left(z_{n_{i}}-\mu_{n_{i}} \Phi\left(z_{n_{i}}\right)-t_{n_{i}}\right)\right\rangle \\
& =\left\langle y-t_{n_{i}}, \Phi(y)-\Phi\left(z_{n_{i}}\right)\right\rangle+\left\langle y-t_{n_{i}}, \frac{z_{n_{i}}-t_{n_{i}}}{\mu_{n_{i}}}\right\rangle \\
& \geq \eta\left\|\Phi(y)-\Phi\left(t_{n_{i}}\right)\right\|^{2}+\left\langle y-t_{n_{i}}, \Phi\left(t_{n_{i}}\right)-\Phi\left(z_{n_{i}}\right)\right\rangle+\left\langle y-t_{n_{i}}, \frac{z_{n_{i}}-t_{n_{i}}}{\mu_{n_{i}}}\right\rangle \\
& \geq\left\langle y-t_{n_{i}}, \Phi\left(t_{n_{i}}\right)-\Phi\left(z_{n_{i}}\right)\right\rangle+\left\langle y-t_{n_{i}}, \frac{z_{n_{i}}-t_{n_{i}}}{\mu_{n_{i}}}\right\rangle .
\end{aligned}
$$


Observe that

$$
\begin{aligned}
& \left|\left\langle y-t_{n_{i}}, \Phi\left(t_{n_{i}}\right)-\Phi\left(z_{n_{i}}\right)\right\rangle+\left\langle y-t_{n_{i}}, \frac{z_{n_{i}}-t_{n_{i}}}{\mu_{n_{i}}}\right\rangle\right| \\
& \leq\left\|y-t_{n_{i}}\right\|\left\|\Phi\left(t_{n_{i}}\right)-\Phi\left(z_{n_{i}}\right)\right\|+\frac{1}{\mu_{n_{i}}}\left\|y-t_{n_{i}}\right\|\left\|z_{n_{i}}-t_{n_{i}}\right\| \\
& \leq \frac{1}{\eta}\left\|y-t_{n_{i}}\right\|\left\|t_{n_{i}}-z_{n_{i}}\right\|+\frac{1}{\epsilon}\left\|y-t_{n_{i}}\right\|\left\|z_{n_{i}}-t_{n_{i}}\right\| \\
& =\left(\frac{1}{\eta}+\frac{1}{\epsilon}\right)\left\|y-t_{n_{i}}\right\|\left\|z_{n_{i}}-t_{n_{i}}\right\| .
\end{aligned}
$$

By $\left\|x_{n}-z_{n}\right\| \rightarrow 0$ and $\left\|x_{n}-t_{n}\right\| \rightarrow 0$, we have $\left\|z_{n}-t_{n}\right\| \rightarrow 0$. Then

$$
\lim _{i \rightarrow \infty}\left|\left\langle y-t_{n_{i}}, \Phi\left(t_{n_{i}}\right)-\Phi\left(z_{n_{i}}\right)\right\rangle+\left\langle y-t_{n_{i}}, \frac{z_{n_{i}}-t_{n_{i}}}{\mu_{n_{i}}}\right\rangle\right|=0 .
$$

Let $i \rightarrow \infty$, from (3.22) we get

$$
\langle y-u, g-0\rangle=\langle y-u, g\rangle \geq 0 .
$$

This implies that $0 \in \Phi(u)+M u$. Hence $u \in \operatorname{VI}(C, \Phi, M)$. Therefore,

$$
u \in \mathcal{F}=\mathcal{F}(S) \cap \Omega \cap \operatorname{VI}(C, \Phi, M) .
$$

Step 5. We show that $x_{n} \rightarrow x^{*}$, where $x^{*}=P_{\mathcal{F}} x_{0}$.

Indeed, from $x^{*}=P_{\mathcal{F} x_{0}}, u \in \mathcal{F}=\mathcal{F}(S) \cap \Omega \cap \operatorname{VI}(C, \Phi, M)$ and (3.9), we have

$$
\left\|x^{*}-x_{0}\right\| \leq\left\|u-x_{0}\right\| \leq \liminf _{i \rightarrow \infty}\left\|x_{n_{i}}-x_{0}\right\| \leq \limsup _{i \rightarrow \infty}\left\|x_{n_{i}}-x_{0}\right\| \leq\left\|x^{*}-x_{0}\right\| .
$$

Then

$$
\lim _{i \rightarrow \infty}\left\|x_{n_{i}}-x_{0}\right\|=\left\|u-x_{0}\right\|
$$

From $x_{n_{i}}-x_{0} \rightarrow u-x_{0}$ and the Kadec-Klee property of $H$, we have $x_{n_{i}}-x_{0} \rightarrow u-x_{0}$, and hence $x_{n_{i}} \rightarrow u$. Since $x_{n_{i}}=P_{C_{n_{i}}} x_{0}$ and $x^{*} \in \mathcal{F} \subset C_{n_{i}}$, we have

$$
-\left\|x^{*}-x_{n_{i}}\right\|^{2}=\left\langle x^{*}-x_{n_{i}}, x_{n_{i}}-x_{0}\right\rangle+\left\langle x^{*}-x_{n_{i}}, x_{0}-x^{*}\right\rangle \geq\left\langle x^{*}-x_{n_{i}}, x_{0}-x^{*}\right\rangle .
$$

Let $i \rightarrow \infty$, by $u \in \mathcal{F}$ and $x^{*}=P_{\mathcal{F}} x_{0}$, we have

$$
-\left\|x^{*}-u\right\|^{2} \geq\left\langle x^{*}-u, x_{0}-x^{*}\right\rangle \geq 0 .
$$

Hence $u=x^{*}$, which implies that $x_{n} \rightarrow x^{*}$. This completes the proof. 


\section{Applications}

From Theorem 3.1, we can obtain the following theorems.

Theorem 4.1 Let $C$ be a nonempty closed convex subset of a real Hilbert space $H$. For the minimization problem (1.4), assume that $f$ is (Frechet) differentiable and the gradient $\nabla f$ is a $\rho$-inverse-strongly monotone mapping. Let $S: C \rightarrow C$ be a $k$-strictly pseudo-contractive mapping such that $\mathcal{F}=\mathcal{F}(S) \cap \Omega \neq \emptyset$. Pick any $x_{0} \in C$ and set $C_{0}=C$. Let $\left\{x_{n}\right\} \subset C$ be a sequence generated by

$$
\left\{\begin{array}{l}
y_{n}=P_{C}\left(x_{n}-\lambda_{n} \nabla f\left(x_{n}\right)\right), \\
z_{n}=P_{C}\left(x_{n}-\lambda_{n} \nabla f\left(y_{n}\right)\right), \\
w_{n}=\alpha_{n} z_{n}+\left(1-\alpha_{n}\right) S z_{n}, \\
C_{n+1}=\left\{w \in C_{n}:\left\|w_{n}-w\right\| \leq\left\|x_{n}-w\right\|\right\}, \\
x_{n+1}=P_{C_{n+1}} x_{0}, \quad n \geq 0,
\end{array}\right.
$$

where the following conditions hold:

(i) $0<\liminf _{n \rightarrow \infty} \lambda_{n} \leq \limsup _{n \rightarrow \infty} \lambda_{n}<\rho$;

(ii) $k<\liminf _{n \rightarrow \infty} \alpha_{n} \leq \limsup _{n \rightarrow \infty} \alpha_{n}<1$.

Then the sequence $\left\{x_{n}\right\}$ converges strongly to $P_{\mathcal{F}} x_{0}$.

Proof Let $\Phi=M=0$ in Theorem 3.1, we have $\operatorname{VI}(C, 0,0)=C$ and $\mathcal{F}=\mathcal{F}(S) \cap \Omega \cap$ $\operatorname{VI}(C, 0,0)=\mathcal{F}(S) \cap \Omega$. Let $\eta$ be any positive number in the interval $(0, \infty)$ and take any sequence $\left\{\mu_{n}\right\} \subset[\epsilon, 2 \eta]$ for some $\epsilon \in(0,2 \eta]$. In addition, we have

$$
t_{n}=J_{M, \mu_{n}}\left(z_{n}-\mu_{n} \Phi\left(z_{n}\right)\right)=\left(I+\mu_{n} M\right)^{-1} z_{n}=z_{n}
$$

Therefore, by Theorem 3.1 we obtain the expected result.

Theorem 4.2 Let $C$ be a nonempty closed convex subset of a real Hilbert space H. Let $S: C \rightarrow C$ be a nonexpansive mapping such that $\mathcal{F}(S) \neq \emptyset$. Pick any $x_{0} \in C$ and set $C_{0}=C$. Let $\left\{x_{n}\right\} \subset C$ be a sequence generated by

$$
\left\{\begin{array}{l}
w_{n}=\alpha_{n} x_{n}+\left(1-\alpha_{n}\right) S x_{n}, \\
C_{n+1}=\left\{w \in C_{n}:\left\|w_{n}-w\right\| \leq\left\|x_{n}-w\right\|\right\}, \\
x_{n+1}=P_{C_{n+1}} x_{0}, \quad n \geq 0,
\end{array}\right.
$$

where the following condition holds: $k<\liminf _{n \rightarrow \infty} \alpha_{n} \leq \limsup _{n \rightarrow \infty} \alpha_{n}<1$. Then the sequence $\left\{x_{n}\right\}$ converges strongly to $P_{\mathcal{F}} x_{0}$.

Proof Let $\nabla f=\Phi=M=0$ and $k=0$ in Theorem 3.1. Let $\rho, \eta$ be any positive number in the interval $(0, \infty)$. Take any sequence $\left\{\lambda_{n}\right\}$ which satisfies $0<\liminf _{n \rightarrow \infty} \lambda_{n} \leq$ $\limsup _{n \rightarrow \infty} \lambda_{n}<\rho$ and take any sequence $\left\{\mu_{n}\right\} \subset[\epsilon, 2 \eta]$ for some $\epsilon \in(0,2 \eta]$. In this case, we have

$$
\left\{\begin{array}{l}
y_{n}=P_{C}\left(x_{n}-\lambda_{n} \nabla f\left(x_{n}\right)\right)=x_{n}, \\
z_{n}=P_{C}\left(x_{n}-\lambda_{n} \nabla f\left(y_{n}\right)\right)=x_{n}, \\
t_{n}=J_{M, \mu_{n}}\left(z_{n}-\mu_{n} \Phi\left(z_{n}\right)\right)=z_{n}, \\
w_{n}=\alpha_{n} t_{n}+\left(1-\alpha_{n}\right) S t_{n}=\alpha_{n} x_{n}+\left(1-\alpha_{n}\right) S x_{n} .
\end{array}\right.
$$

Therefore, by Theorem 3.1 we obtain the expected result. 
Theorem 4.3 Let C be a nonempty closed convex subset of a real Hilbert space H. For the minimization problem (1.4), assume that $f$ is (Frechet) differentiable and the gradient $\nabla f$ is a $\rho$-inverse-strongly monotone mapping. Let $\Gamma: C \rightarrow C$ be $\gamma$-strictly pseudo-contractive and let $S: C \rightarrow C$ be a k-strictly pseudo-contractive mapping such that $\mathcal{F}=\mathcal{F}(S) \cap \Omega \cap$ $\mathcal{F}(\Gamma) \neq \emptyset$. Pick any $x_{0} \in C$ and set $C_{0}=C$. Let $\left\{x_{n}\right\} \subset C$ be a sequence generated by

$$
\left\{\begin{array}{l}
y_{n}=P_{C}\left(x_{n}-\lambda_{n} \nabla f\left(x_{n}\right)\right), \\
z_{n}=P_{C}\left(x_{n}-\lambda_{n} \nabla f\left(y_{n}\right)\right), \\
t_{n}=\left(1-\mu_{n}\right) z_{n}+\mu_{n} \Gamma\left(z_{n}\right), \\
w_{n}=\alpha_{n} t_{n}+\left(1-\alpha_{n}\right) S t_{n}, \\
C_{n+1}=\left\{w \in C_{n}:\left\|w_{n}-w\right\| \leq\left\|x_{n}-w\right\|\right\}, \\
x_{n+1}=P_{C_{n+1}} x_{0}, \quad n \geq 0,
\end{array}\right.
$$

where the following conditions hold:

(i) $0<\liminf _{n \rightarrow \infty} \lambda_{n} \leq \lim \sup _{n \rightarrow \infty} \lambda_{n}<\rho$;

(ii) $\epsilon \leq \mu_{n} \leq 1-\gamma$ for some $\epsilon \in(0,1-\gamma]$;

(iii) $k<\liminf _{n \rightarrow \infty} \alpha_{n} \leq \limsup _{n \rightarrow \infty} \alpha_{n}<1$.

Then the sequence $\left\{x_{n}\right\}$ converges strongly to $P_{\mathcal{F}} x_{0}$.

Proof Let $\Phi=I-\Gamma$ and $M=0$ in Theorem 3.1, then we have that $\Phi$ is $\eta$-inverse strongly monotone with $\eta=\frac{1-\gamma}{2}$. Now, we show that $\operatorname{VI}(C, \Phi, M)=\mathcal{F}(\Gamma)$. In fact, since $\Phi=I-\Gamma$ and $M=0$, we obtain

$$
\begin{aligned}
0 \in \mathrm{VI}(C, \Phi, M) & \Leftrightarrow \quad 0 \in \Phi(u)+M u \\
& \Leftrightarrow \quad 0=\Phi(u) \\
& \Leftrightarrow \quad 0=u-\Gamma(u) \\
& \Leftrightarrow \quad u \in \mathcal{F}(\Gamma) .
\end{aligned}
$$

Thus,

$$
\mathcal{F}=\mathcal{F}(S) \cap \Omega \cap \operatorname{VI}(C, \Phi, M)=\mathcal{F}(S) \cap \Omega \cap \mathcal{F}(\Gamma) .
$$

Note that $\mu_{n} \in[\epsilon, 1-\gamma] \subset[0,1]$, hence $\left(1-\mu_{n}\right) z_{n}+\mu_{n} \Gamma\left(z_{n}\right) \in C$. In this case, we have

$$
\begin{aligned}
t_{n} & =J_{M, \mu_{n}}\left(z_{n}-\mu_{n} \Phi\left(z_{n}\right)\right) \\
& =\left(I+\mu_{n} M\right)^{-1}\left(z_{n}-\mu_{n} \Phi\left(z_{n}\right)\right) \\
& =z_{n}-\mu_{n} \Phi\left(z_{n}\right)=z_{n}-\mu_{n}(I-\Gamma)\left(z_{n}\right) \\
& =\left(1-\mu_{n}\right) z_{n}+\mu_{n} \Gamma\left(z_{n}\right) .
\end{aligned}
$$

Therefore, by Theorem 3.1 we obtain the expected result. 
Authors' contributions

All authors contributed equally to the writing of this paper. All authors read and approved the final manuscript.

\section{Acknowledgements}

Changfeng Ma is grateful for the hospitality and support during his research at Chern Mathematics Institute in Nankai University in June 2013. The project is supported by the National Natural Science Foundation of China (11071041), Fujian Natural Science Foundation (2013J01006) and R\&D of Key Instruments and Technologies for Deep Resources Prospecting (the National R\&D Projects for Key Scientific Instruments) under grant number ZDYZ2012-1-02-04.

\section{Received: 8 October 2013 Accepted: 19 February 2014 Published: 04 Mar 2014}

\section{References}

1. Hartman, P, Stampacchia, G: On some nonlinear elliptic differential equations. Acta Math. 115, 271-310 (1966)

2. Hlaváček, I, Haslinger, J, Nečas, J, Lovišek, J: Solution of Variational Inequalities in Mechanics. Applied Mathematical Science, vol. 66. Springer, New York (1988)

3. Fukushima, M: Equivalent differentiable optimization problems and descent methods for asymmetric variational inequality problems. Math. Program. 53, 99-110 (1992)

4. Dafermos, S: Traffic equilibrium and variational inequalities. Transp. Sci. 14, $42-54$ (1980)

5. Zhang, SS, Lee, JHW, Chan, CK: Algorithms of common solutions to quasi variational inclusion and fixed point problems. Appl. Math. Mech. 29, 571-581 (2008)

6. Zhang, SS, Kim, JK, Kim, KH: On the existence and iterative approximation problems of solutions for set-valued variational inclusions in Banach spaces. J. Math. Anal. Appl. 268, 89-108 (2002)

7. Zhang, SS: Set-valued variational inclusions in Banach spaces. J. Math. Anal. Appl. 248, 438-454 (2000)

8. Huang, NJ: Generalized nonlinear variational inclusions with noncompact valued mappings. Appl. Math. Lett. 9, 25-29 (1996)

9. Noor, MA: Implicit resolvent dynamical systems for quasi variational inclusions. J. Math. Anal. Appl. 269, 216-226 (2002)

10. Noor, MA: Three-step iterative algorithms for multivalued quasi variational inclusions. J. Math. Anal. Appl. 255 589-604 (2001)

11. Noor, MA, Noor, KI: Sensitivity analysis for quasi-variational inclusions. J. Math. Anal. Appl. 236, $290-299$ (1999)

12. Verma, RU: Approximation solvability of a class of nonlinear set-valued variational inclusions involving $(A, \eta)$-monotone mappings. J. Math. Anal. Appl. 337, 969-975 (2008)

13. Verma, RU: A-monotonicity and its role in nonlinear variational inclusions. J. Optim. Theory Appl. 129, 457-467 (2006)

14. Verma, RU: General nonlinear variational inclusion problems involving A-monotone mappings. Appl. Math. Lett. 19, 960-963 (2006)

15. Butnariu, D, Censor, Y, Gurfil, P, Hadar, E: On the behavior of subgradient projections methods for convex feasibility problem in Euclidean spaces. SIAM J. Optim. 19, 786-807 (2008)

16. Maruster, S, Popirlan, C: On the Mann-type iteration and the convex feasibility problem. J. Comput. Appl. Math. 212, 390-396 (2008)

17. Xu, HK: A variable Krasnosel'skii-Mann algorithm and the multiple-set split feasibility problem. Inverse Probl. 22 2021-2034 (2006)

18. Youla, D: Mathematical theory of image restoration by the method of convex projections. In: Stark, H (ed.) Image Recovery Theory and Applications. Academic Press, Orlando (1987)

19. Ke, YF, Ma, CF: A new relaxed extragradient-like algorithm for approaching common solutions of generalized mixed equilibrium problems, a more general system of variational inequalities and a fixed point problem. Fixed Point Theory Appl. 2013, 126 (2013)

20. Ke, YF, Ma, CF: The convergence analysis of the projection methods for a system of generalized relaxed cocoercive variational inequalities in Hilbert spaces. Fixed Point Theory Appl. 2013, 189 (2013)

21. Zegeye, H, Shahzad, N, Alghamdi, MA: Convergence of Ishikawa's iteration method for pseudocontractive mappings. Nonlinear Anal. 74, 7304-7311 (2011)

22. Tang, YC, Peng, JG, Liu, LW: Strong convergence theorem for pseudo-contractive mappings in Hilbert spaces. Nonlinear Anal. 74, 380-385 (2011)

23. Scherzer, O: Convergence criteria of iterative methods based on Landweber iteration for solving nonlinear problems. J. Math. Anal. Appl. 194, 911-933 (1991)

24. Ceng, LC, Chou, CY: On the relaxed hybrid-extragradient method for solving constrained convex minimization problems in Hilbert spaces. Taiwan. J. Math. 17, 911-936 (2013)

25. Brezis, H: Opérateur maximaux monotones et semigroupes de contractions dans les espaces de Hilbert. North-Holland, Amsterdam (1973)

26. Acedo, GL, Xu, HK: Iterative methods for strict pseudo-contractions in Hilbert spaces. Nonlinear Anal. 67, 2258-2271 (2007)

27. Rockafellar, RT: On the maximality of sums of nonlinear monotone operators. Trans. Am. Math. Soc. 149, 75-88 (1970)

28. Su, M, Xu, HK: Remarks on the gradient-projection algorithm. J. Nonlinear Anal. Optim. 1, 35-43 (2010)

10.1186/1687-1812-2014-54

Cite this article as: Ke and Ma: Iterative algorithm of common solutions for a constrained convex minimization problem, a quasi-variational inclusion problem and the fixed point problem of a strictly pseudo-contractive mapping. Fixed Point Theory and Applications 2014, 2014:54 